\title{
The Star Clusters in the Starburst Irregular Galaxy NGC 1569
}

\author{
Deidre A. Hunter \\ Lowell Observatory, 1400 West Mars Hill Road, Flagstaff, Arizona 86001 USA; \\ dah@lowell.edu \\ Robert W. O'Connell \\ University of Virginia, Department of Astronomy, PO Box 3818, Charlottesville, Virginia \\ 22903-0818 USA; \\ rwo@virginia.edu \\ J. S. Gallagher \\ Washburn Observatory, University of Wisconsin, 475 N. Charter St., Madison, Wisconsin 53706 \\ USA; \\ jsg@astro.wisc.edu \\ and \\ Tammy A. Smecker-Hane \\ University of California, Department of Physics and Astronomy, 4129 Reines Hall, Irvine, \\ California 92697-4575 USA; \\ smecker@carina.ps.uci.edu
}

\begin{abstract}
We examine star clusters in the irregular, starburst galaxy NGC 1569 from HST images taken with filters F336W, F555W, and F814W. In addition to the two super star clusters that are well known, we identify 45 other clusters that are compact but resolved. Integrated UVI colors of the clusters span a large range, and comparison with coeval evolutionary models suggest that the ages range from 2-3 Myrs to 1 Gyr. Most of the clusters have colors consistent with ages of $\leq 30$ Myrs placing them at the tail end of the recent burst of star formation.

We examine the radial surface brightness profiles of four of the clusters, and fit King models to three of them. The colors of the clusters are approximately constant with radius. The four clusters have half-light radii and core radii that are in the range observed in present-day globular clusters in our Galaxy. However, they are somewhat less concentrated that the average globular. The two well-known super star clusters have luminosities, and one has a known mass, that are comparable to those of typical
\end{abstract}

\footnotetext{
${ }^{1}$ Based on observations with the NASA/ESA Hubble Space Telescope, obtained at the Space Telescope Science Institute, which is operated by AURA, Inc., under NASA contract NAS 5-26555.
} 
globular clusters. The other two clusters, and likely numerous others in the sample, are similar to a small globular cluster and to R136 in the LMC. The conditions that produced the recent starburst, therefore, have also been those necessary for producing compact, bright star clusters.

We examine resolved stars in the outer parts of the super star clusters. We find that cluster A contains many bright blue stars. Some of the blue stars are bright enough to be evolved massive stars. There is also a small population of red supergiants. Components A1 and A2 within cluster A have similar colors and a two-dimensional color map does not offer evidence that one component is dominated by red supergiants and the other not. The contradiction of the presence of red supergiants with Wolf-Rayet stars may instead not be a contradiction at all since there coexistence in a coeval population is not inconsistent with the evolution of massive stars. Alternatively, there may be a small age spread of several Myrs within cluster A. The stars that we resolve around cluster $\mathrm{B}$, on the other hand, contain a small population of more normal blue massive stars and a large population of red supergiants. The presence of the red supergiants is consistent with the view that cluster B is in its red supergiant phase. The presence of the red supergiant stars in clusters A and B is also verified in near-infrared spectra where we find strong stellar CO absorption features. The various age indicators are consistent with a picture in which cluster B is of order 10-20 Myrs old, and cluster $\mathrm{A}$ is $\geq 4-5$ Myrs old. The timescale to form the holes seen in $\mathrm{H} \alpha$ and $\mathrm{HI}$ is comparable to the age of cluster B.

Subject headings: galaxies: irregular — galaxies: star formation — galaxies: individual: NGC 1569 - galaxies: star clusters

\section{Introduction}

With the Hubble Space Telescope (HST) people are finding increasing numbers of super star clusters (see, for example, Holtzman et al. 1992; Whitmore et al. 1993; Conti \& Vacca 1994; Hunter et al. 1994; O'Connell et al. 1994, 1995; Barth et al. 1995). These are compact, luminous star clusters that have sizes and luminosities (when scaled to a common age) that make them comparable to globular clusters, the most massive star clusters known. However, unlike globular clusters, the super star clusters being found today are often young, in some cases as young as a few Myrs. Therefore, these super star clusters are invaluable in providing us with a unique window on the early stages, evolution, and conditions necessary to form globular-type clusters. They also probe the star formation process at one of its extremes.

Young super star clusters as massive as globular clusters are not common in normal disk galaxies, but there are several of these clusters within a few Mpc of the Milky Way. Because of their proximity, these clusters are the best examples for investigating the details of the clusters 
themselves. The closest example of a young massive, compact star cluster is R136, at the center of the 30 Doradus nebula in the Large Magellanic Cloud (LMC). Within a cluster radius of 4.7 pc, R136 contains 300 times the concentration of luminous stars in a typical OB association (O'Connell et al. 1994, Hunter 1995). R136 is unique among the globular-like clusters in that it can be resolved into individual stars and one can investigate the mass function resulting from such a concentrated star-forming event. Nevertheless, R136 is several magnitudes fainter than other super star clusters when normalized to the same age. R136 has an integrated V-band magnitude of only -11 .

Beyond the LMC, the next closest known super star clusters are among the most extreme in terms of luminosity. These are clusters A and B located in the nearby peculiar irregular galaxy NGC 1569 (Ables 1968). These clusters are so compact that they appear stellar in ground-based images with good seeing, and their nature was controversial (Arp \& Sandage 1985). However, HST Cycle 1 images resolved these objects, proving them to be star clusters within NGC 1569 with absolute V-band magnitudes of -14 and -13 (O'Connell et al. 1994).

These super star clusters are resident in the central region of a galaxy that itself is very unusual. NGC 1569 has recently undergone a true wide-scale burst of star formation involving most of the optical galaxy (de Vaucouleurs, de Vaucouleurs, \& Pence 1974; Hodge 1974; Gallagher, Hunter, \& Tutukov 1984; Israel 1988; Vallenari \& Bomans 1996; Greggio et al. 1998). The galaxy still today has substantial on-going star formation as seen by the presence of bright H iI regions. In addition Della Ceca et al. (1996) have detected hard X-rays from supernova remnants and binaries associated with the center of the galaxy and soft, diffuse X-rays extending along the minor axis of the galaxy (see also Heckman et al. 1995), both consequences of the intense recent star formation. Furthermore, there are large filaments of ionized gas visible in $\mathrm{H} \alpha$ that extend to $1.9 \mathrm{kpc}$ from the center of the galaxy (Waller 1991; Hunter, Hawley, \& Gallagher 1993; Hunter \& Gallagher 1997) and a complex velocity field in the ionized gas (Tomita, Ohta, \& Sait_o 1994). Stil \& Israel (1998) have detected a $7 \times 10^{6} \mathrm{M}_{\odot}$ HI cloud at $5 \mathrm{kpc}$ from NGC 1569 and the hint of a bridge in HI connecting them. The suggestion is that interaction with this HI cloud is responsible for the recent high level of star formation. Furthermore, it is perhaps a consequence of these unusual circumstances that this galaxy has made two of the rare super star clusters.

In order to examine the structure of these super star clusters in NGC 1569, we obtained post-refurbishment images of the galaxy with HST. With these data we intended to test the suggestion that the super star clusters in NGC 1569 are young versions of globular clusters. Examination of the clusters from Cycle 1 HST images and early post-refurbishment data have already revealed that cluster A has two brightness peaks and so is not as symmetrical as globular clusters are today (O'Connell et al. 1994, De Marchi et al. 1997). What our data contribute to this continuing discussion, besides the higher resolution compared to the earliest HST images, is a deeper, and hence higher signal-to-noise, look at these clusters and I-band images that are sensitive to the red supergiant star population of the clusters. In addition we examine the other compact clusters in the galaxy to learn about the ensemble of star clusters formed in this unusual 
star formation episode in NGC 1569.

\section{The Observations and Data Reduction}

\subsection{The $H S T$ Data}

The center of NGC 1569 was imaged with the Wide Field and Planetary Camera 2 (WFPC2) on HST on 1998 October 21. The galaxy was centered on the PC for maximum resolution of star clusters A and B: $0.04555^{\prime \prime}$ per pixel which is $0.55 \mathrm{pc}$ at the galaxy. The galaxy was imaged through

filters F336W, F555W, F814W, and F656N. Exposures through F555W and F814W consisted of a series of three integration times - short, medium, and long. In the longer exposures the centers of clusters A and B are saturated. For the longer exposures there are multiple exposures in order to improve signal-to-noise and remove cosmic rays. The list of observations are given in Table 1 .

Basic data reduction steps were done by the Space Telescope Science Institute "pipeline" processing system. We produced a nebular emission image by combining the medium-exposure F555W and F814W images, shifting, scaling, and subtracting from the F656N image to remove the stellar continuum. We then subtracted nebular emission from the F555W and F814W images, as needed, using a scaled and shifted $\mathrm{H} \alpha$ emission image. A mosaic of the CCD frames is shown in Figure 1. A false-color combination of F555W, F814W, and F656N is shown in Figure 2.

We measured the brightnesses of field stars in the galaxy using the crowded star photometry package DAOPHOT (Stetson 1987) as implemented in the Image Reduction and Analysis Facility (IRAF). Here we will discuss the resolved stars in and near the star clusters A and B; we will discuss analysis of the field star population itself in another paper.

We measured integrated photometry of star clusters using simulated aperture photometry. We calibrated the photometry using the zero point constants given by Holtzman et al. (1995b) in their Table 9. We also converted the F336W, F555W, and F814W photometry to the Johnson/Cousins UVI system using the conversions of Holtzman et al. The instrumental magnitudes were corrected for reddening and the red leak in F336W, as discussed below, before converting to UVI, as discussed by Holtzman et al.

\subsection{Near-IR Spectroscopy}

We obtained longslit near-infrared spectra of clusters A and B in order to examine the stellar CO features, which are an age diagnostic through sensitivity to the presence of red supergiants. The spectra were obtained over 3 nights in 1995 December with the Ohio State Infrared Imager/Spectrometer (OSIRIS) on the $1.8 \mathrm{~m}$ Perkins telescope. The spectra cover 21900 $\AA$ to $23900 \AA$ at $8 \AA$ per pixel. The clusters were observed in alternating positions on the slit, 
$10^{\prime \prime}$ apart. One position was used as a sky observation for the other. We also alternated a 25-70 minute series of observations of a cluster with a pair of observations of HR 1440, an A1V star without the CO spectral features, chosen to be close to NGC 1569 in airmass. We also observed a series of bright stars of various spectral types for comparison to the clusters.

We corrected the data for non-linearities using dark and flat field observations. The flat sequence consisted of a series of increasing exposure times intertwined with 1 second flat exposures to remove drifts in the lamps. Dark observations preceeded and followed the flat field observations. Regular dome flats were also taken and the data were flat fielded. Sky frames were subtracted from object frames, one-dimensional spectra were extracted, and subgroups of spectra were combined. Night sky lines in spectra with the sky not subtracted were used to determine the wavelength scale and linearize along the dispersion axis. The night sky lines were identified using a line list provided by M. Hanson (private communication) which came originally from C. Kulesa (private communication to M. Hanson). The cluster spectra were divided by the nearest spectrum of HR 1440. in order to remove the telluric absorption. For the cluster spectra the continuum was also fit and the spectra divided by the fit.

\section{Data Analysis Issues}

\subsection{Reddening}

The reddening to and within NGC 1569 has been estimated by various methods. The reddening in the Milky Way in the direction of NGC $1569 \mathrm{E}(\mathrm{B}-\mathrm{V})_{f}$ is estimated to be 0.51 by Burstein \& Heiles (1984) from the column density of HI. Israel (1988) measured a total reddening $\mathrm{E}(\mathrm{B}-\mathrm{V})_{t}$ of $0.56 \pm 0.10$ from ANS ultraviolet data. Devost, Roy, \& Drissen (1997) obtained optical emission spectra of the ionized gas in NGC 1569 and concluded that foreground $\mathrm{E}(\mathrm{B}-\mathrm{V})_{f}$ is 0.52 and internal $\mathrm{E}(\mathrm{B}-\mathrm{V})_{i}$ is 0.21 for $\mathrm{R}=3.1$. Using optical spectra Kobulnicky \& Skillman (1997) examined the variation of reddening within the galaxy and found that $\mathrm{c}(\mathrm{H} \beta)$ varies from 0.8 to 1.2 with an average of $0.97 \pm 0.07$. The average translates into an $\mathrm{E}(\mathrm{B}-\mathrm{V})_{t}$ of 0.63 for $\mathrm{R}=3.1$.

Figure 2 clearly shows that there is ionized, and hence probably neutral gas and dust, distributed non-uniformly around the galaxy. From this figure one can see that clusters A and B themselves are sitting in a large hole in the ionized gas and Israel \& van Driel (1990) state that they are sitting in holes in the HI gas as well (see also Swaters 1999). Thus, the extinctions to these clusters are likely to be at the low end of the range in NGC 1569. However, other clusters are embedded in $\mathrm{H}$ II regions and are likely to have extinctions at the high end of the range. Nevertheless, according to Kobulnicky \& Skillman (1997), the total range in $\mathrm{E}(\mathrm{B}-\mathrm{V})_{t}$ is only 0.56 to 0.71 , where 0.51 of that is foreground Milky Way reddening.

We have used Figure 2 to classify the clusters in 3 extinction bins-heavy, medium, and light, according to how much ionized gas is present in the immediate vicinity of the cluster. Clusters 
6,41 , and 44 are considered to be heavily internally extincted and $\mathrm{E}(\mathrm{B}-\mathrm{V})_{t}$ is taken to be 0.71 for these. Clusters 4, 7, 8, 9, 10, 39, 40, and 42 are considered to have medium extinction and $\mathrm{E}(\mathrm{B}-\mathrm{V})_{t}$ is taken to be 0.63 . All other clusters are taken to be lightly internally extincted and an $\mathrm{E}(\mathrm{B}-\mathrm{V})_{t}$ of 0.56 is adopted. Although this manner of determining the reddening is rough, the uncertainty in any adopted $\mathrm{E}(\mathrm{B}-\mathrm{V})$ can be no more than \pm 0.15 magnitude, the total range in $\mathrm{E}(\mathrm{B}-\mathrm{V})$ measured from spectroscopy. We use the Cardelli, Clayton, \& Mathis (1989) extinction curve and an $\mathrm{A}_{V} / \mathrm{E}(\mathrm{B}-\mathrm{V})$ of 3.1. (Although NGC 1569 is of low metallicity compared to the Milky Way, this has small effect on the optical extinction curve [Bouchet et al. 1985]).

Holtzman et al. (1995b) showed that the reddening correction is a function of the spectrum of the object, and at F336W the difference between the extinction of an O6 star and a K5 star can be large. The colors of the clusters cover a large range, comparable to that of the stellar main sequence. Therefore, we have determined a reddening correction that depends on the integrated colors of the clusters. To determine the extinction as a function of the observed colors of objects, we used the tools in the Space Telescope Science Data Analysis System (STSDAS) to simulate the throughput of the telescope plus filters for various blackbodies reddened by $\mathrm{E}(\mathrm{B}-\mathrm{V})_{t}$ 's of 0.56 , 0.63 , and 0.71 . The extinction corrections for O6 and K5 type spectra are those given by Holtzman et al. in their Table 12; we have simply used STSDAS simulations to determine extinctions for effective temperatures in between these. The cluster photometry was then corrected for extinction according to its observed $\mathrm{F} 555 \mathrm{~W}-\mathrm{F} 814 \mathrm{~W}$ color and its $\mathrm{E}(\mathrm{B}-\mathrm{V})_{t}$ category.

\section{2. $\quad$ Red Leak in F336W}

Since we wish to examine the F336W-F555W color of clusters, we need to consider the red leak in the F336W filter. Not only are many of these clusters intrinsically red, but they are also highly reddened from foreground extinction. This means that red photons could contribute significantly to the counts in the F336W filter. To determine the red leak as a function of the observed F555W-F814W color, we used the simulations in STSDAS and blackbody curves reddened by $\mathrm{E}(\mathrm{B}-\mathrm{V})_{t}$ 's of $0.56,0.63$, and 0.71 . The red leak was taken to be any flux contribution

from $\geq 4000 \AA$, after the definition of Holtzman et al. (1995b). The F336W cluster photometry was corrected for the red leak based on its observed F555W-F814W color and its reddening category. For an especially red cluster with an observed $\mathrm{F} 555 \mathrm{~W}-\mathrm{F} 814 \mathrm{~W}$ of 1.5 and an $\mathrm{E}(\mathrm{B}-\mathrm{V})_{t}$ of 0.71 , the contribution of the red leak to the F336W magnitude is 0.06 magnitudes, but the correction increases rapidly for redder observed colors.

\subsection{Distance to the Galaxy}

Israel (1988) used a distance to NGC 1569 of 2.2 \pm 0.6 Mpc. From WFPC1 observations O'Connell et al. (1994) measured the brightnesses of the brightest stars in NGC 1569, and, 
assuming an $\mathrm{E}(\mathrm{B}-\mathrm{V})_{t}$ of 0.56 from Israel (1988), determined a distance of $2.5 \pm 0.5 \mathrm{Mpc}$. Greggio et al. (1998) examined the color magnitude diagram of the galaxy from WFPC2 images and adopted an $\mathrm{E}(\mathrm{B}-\mathrm{V})_{t}$ of 0.56 and distance of $2.2 \mathrm{Mpc}$ without apparent distress to the analysis of the stellar population. In this paper we will adopt a distance of $2.5 \mathrm{Mpc}$.

\section{Identification of Clusters}

Clusters A and B are the most spectacular star clusters in NGC 1569, but they are not the only ones. We have examined the WFPC2 images for other compact, but less luminous, clusters. We have included as a cluster any compact object that was resolved compared to an isolated star profile. Contamination of the sample by background galaxies is possible although WF2 and WF3, which include little of NGC 1569, contain few background galaxies and no objects outside of NGC 1569 that fit our criteria.

Generally, we have not included looser, and spatially bigger, OB associations in our list of clusters. The reason for this is that the galaxy is really one big OB association and separating one from another is not feasible. Also, we are interested in the less common compact clusters for comparison with clusters A and B. However, we did include one OB association, cluster number 48 on WF4. This OB association is discussed by Drissen \& Roy (1994) who discovered a ring nebula surrounding the cluster and broad stellar emission lines that they attribute to a WN star. It is located in the outer part of NGC 1569 and was easy to isolate. It serves as an example of a certified $\mathrm{OB}$ association for comparison to the other clusters.

Because the clusters are so numerous, we could not reasonably continue the identification scheme begun by others with clusters A and B. Therefore, we switched to using numbers as identifiers, but began with number 3, so clusters A and B are also numbers 1 and 2. The clusters are identified in Figure 3, and are listed in Table 2.

Ten of the newly identified clusters are located in the vicinity of super star cluster A, over a region of about $50 \mathrm{pc}$ projected on the sky. Together they may form something akin to a small version of the greater 30 Doradus region in the LMC. 30 Doradus is a kpc-sized area in which star formation has taken place in small units here and there over a timescale of order 10 Myrs (Walborn \& Blades 1997; see also Constellation III, Dolphin \& Hunter 1998). Otherwise the clusters are distributed throughout the galaxy with no apparent pattern.

\section{Integrated Cluster Photometry}

We give integrated photometry of the star clusters in Table 2. The contributions from the sky and background galaxy were measured in an annulus just beyond the integration aperture for each object. The aperture radius is given in Table 2 . 
We show the integrated colors and magnitudes in color-color and color-magnitude diagrams in Figures 1 and 5 . We also include cluster evolutionary tracks from Leitherer et al. (1999). We use their tables for an instantaneous burst of star formation and a Salpeter (1955) stellar initial mass function from 1 to $100 \mathrm{M}_{\odot}$. Time steps of 1 to 9 Myrs in steps of 1 Myrs are marked with an " $\mathrm{x}$ " along this evolutionary track; time steps of 10, 20, and 30 Myrs are marked with open circles. The $\mathrm{M}_{V}$ have been adjusted from the models of $10^{6} \mathrm{M}_{\odot}$ to the mass of $3.3 \times 10^{5} \mathrm{M}_{\odot}$ determined for cluster A by Ho \& Filippenko (1996). For less massive clusters the tracks in Figure 5 would slide vertically to fainter $\mathrm{M}_{V}$.

Kobulnicky \& Skillman (1997) give the oxygen abundance of emission nebulae in NGC 1569 as $8.19 \pm 0.02$ with no evidence for chemical inhomogeneities. This oxygen abundance implies a metallicity $Z$ of approximately 0.004 . One would expect that the metallicity of current $\mathrm{H}$ II regions in NGC 1569 should be a good estimate of the metallicities of the recently formed stars. Therefore, we began with the $Z=0.004$ models of Leitherer et al. (1999). However, we found that the $Z=0.004$ cluster evolutionary tracks did not account very well for the colors of some of the clusters, but that $Z=0.008$ models did. Therefore, in Figures 1 and 5 we include cluster models for both $Z=0.004$ and $Z=0.008$.

In Figures 1 and 5 we see that cluster A has UVI colors that are consistent with an age of order 4-5 Myrs, according to cluster evolutionary models for both metallicities. This is consistent with the detection of the signature of Wolf-Rayet stars in the cluster (Delgado et al. 1997). However, O'Connell et al. (1994) and De Marchi et al. (1997) have shown that cluster A has two peaks in the light distribution, suggesting two sub-clusters. De Marchi et al. also suggest that these two sub-clusters may have different ages, with one being dominated by Wolf-Rayet stars and the other dominated by red supergiants. However, the integrated UVI colors of this cluster do not reflect that and appear to be dominated solely by the younger component.

Many of the other clusters in Figure 1 likewise fall near the locus of the $Z=0.004$ models. The group lying above cluster A in the diagram and slightly blueward of the locus can be explained if we have made a small overcorrection to their extinction.

However, cluster $\mathrm{B}$ and 8 fainter objects are too red in $(\mathrm{V}-\mathrm{I})$ to be consistent with the $Z=0.004$ locus. We believe this is a symptom of an important contribution from red supergiants to the U,V,I light. The colors of the Leitherer et al. (1999) models exhibit strong sensitivity to abundance in the range $Z=0.004$ to $Z=0.008$ for ages $8-16 \mathrm{Myr}$, as shown in Figure 4 , because of the influence of red supergiants at these ages. Unfortunately, as emphasized by Origlia et al. (1999), there is considerable uncertainty about the evolutionary tracks for such phases at subsolar metallicities. What we can say is that cluster B and the other objects in its vicinity in Figure 1 are roughly consistent with the $Z=0.008$ models of Leitherer et al. (1999) at an age betweeen 8 and 15 Myr. The agreement for cluster B would be particularly good if we have overcorrected for extinction by about 0.2 mags in $\mathrm{E}(\mathrm{B}-\mathrm{V})$. However, the $\mathrm{E}(\mathrm{B}-\mathrm{V})$ that we assumed for cluster $\mathrm{B}$ is only 0.05 magnitude above the estimated foreground extinction, so it is unlikely that we have 
overestimated the extinction by very much. An age of 8-15 Myrs, however, is consistent with the CO-band evidence for red supergiants discussed in Section 6.3.

The youngest cluster, occupying the upper left in Figure 4 , is number 48, the OB association in the southeast part of the galaxy. Its colors indicate an age of just 2-3 Myrs. This age is consistent with the upper limit of 5 Myrs placed by Drissen \& Roy (1994) based on their observation of an expanding bubble due to a WN-type evolved massive star. The rest of the clusters, with the exception of the group around cluster B, span the range of colors along the cluster evolutionary sequence.

Part of the scatter seen in Figure 1 is caused by stochastic effects. The latter are discussed and simulated by Girardi \& Bica (1993; see also, Santos \& Frogel 1997; Brocato et al. 1999). Stochastic effects are due to small number statistics in populating the upper masses of the stellar initial mass function; as the few massive stars in small clusters evolve, the status of a single star can have a profound affect on the integrated colors of a small cluster. Girardi and Bica's simulated color-color diagram shows a scatter in UBV colors of several tenths magnitude. This problem affects clusters as old as 1 Gyr as well as young ones (see, for example, Gallagher \& Smith 1999). Brocato et al. further point out the difficulty in correcting for the red leak in HST filters under these circumstances. In addition, differences of several tenths magnitude in colors can be seen between different models depending on the treatment of various evolutionary parameters (see, for example, Brocato et al. 1999). Thus, there is considerable uncertainty in assigning an age to a specific cluster from global colors.

Keeping the observational, stochastic, and modeling uncertainties in mind, we conclude from Figure 1 that the clusters roughly span the full range in ages covered by the evolutionary models from the $\mathrm{OB}$ association, which is the youngest at a few Myrs, to nearly 1 Gyr. However, the distribution is very unlike what would be expected for a uniform distribution of ages. Instead, there is a very strong concentration to ages less than $30 \mathrm{Myr}$, with a subconcentration at 4-6 Myr. Therefore, most of the clusters have been formed in the recent starburst activity of the galaxy. The presence of a few potentially older clusters suggests that the galaxy also formed stars in compact clusters even before the advent of the present starburst.

Clusters A and B are by far the most luminous of the clusters. The rest of the clusters in our sample have $M_{V}$ between -7 and -12 . As shown in Figure 5, the model clusters evolve at about constant $\mathrm{M}_{V}$ for the first $7 \mathrm{Myrs}$, and $\mathrm{M}_{V}$ steadily declines after that, losing several magnitudes by the time the cluster reaches the colors of the reddest clusters in our sample. Therefore, the bluer clusters with $\mathrm{M}_{V}$ near -11 and the redder clusters with $\mathrm{M}_{V}$ near -8 are likely to have had absolute magnitudes of order -11 when they were a few Myrs old. This implies that these clusters are comparable in stellar mass to the compact cluster R136 in the LMC. R136 has an $\mathrm{M}_{V}$ of -11 at an age of 2 Myrs (Hunter et al. 1995, Massey \& Hunter 1998). Since R136 is comparable to a small globular cluster in mass and compactness, it appears that NGC 1569, besides making two very luminous super star clusters, has also made another half dozen or so clusters comparable to 
small globulars as well.

In addition to the more luminous star clusters, however, there are many less luminous clusters. Those with $\mathrm{M}_{V}$ near -7 likely contain $\leq 15$ massive stars, and would be considered more like OB associations in terms of stellar content if they were not so compact in size. The OB association, number 48 , included in the sample of clusters has an $\mathrm{M}_{V}$ of -8.8 and was encircled by an aperture with radius 14 pc. Except for clusters A and B, the other clusters were observed with apertures smaller than this: $0.2-11 \mathrm{pc}$, with an average of $3 \mathrm{pc}$, in radius. Some of these may be comparable to the LMC's populous clusters. For example, the populous cluster NGC 1818 in the LMC has an $\mathrm{M}_{F 555 W, 0}$ of -9.3 now and probably -10.5 at an age of 4 Myrs with a half-light radius of $3.2 \mathrm{pc}$ (Hunter et al. 1997).

Thus, it appears that NGC 1569 in its recent burst of star formation has formed many compact and relatively massive star clusters. These clusters include the extreme super star clusters A and B, as well several clusters comparable to small globular clusters in luminosity and many other comparable to normal OB associations but more compact than is typical. We do not understand what conditions are necessary to produce compact clusters or super star clusters (see, for example, discussion by Dolphin \& Hunter 1998). However, those conditions appear to have been met recently in NGC 1569.

\section{The Super Star Clusters}

From HST Cycle 1 data, the super star clusters A and B were found to have half-light radii $\mathrm{R}_{0.5}$ of 2.2 and $3.0 \mathrm{pc}$, compared to $\mathrm{R}_{0.5}$ of $1-8 \mathrm{pc}$ for today's Milky Way globular clusters (van den Bergh et al. 1991). The integrated V-band magnitudes of the clusters in NGC 1569 are -14 and -13 , corrected for extinction. As shown in Figure 5, both would have had $\mathrm{M}_{V} \sim-14$ at an age of 4 Myrs. By contrast, a typical globular cluster, if it had a Salpeter (1955) stellar initial mass function, would be expected to have had a magnitude of $-13.7 \pm 1.3$ (Harris 1991). Thus, the clusters in NGC 1569 have luminosities and sizes that are comparable to globular clusters that are seen in our Galaxy today, and they are excellent candidates for being true young globulars.

The extreme nature of NGC 1569's super star clusters have long intrigued astronomers, and some properties, particularly their ages, have remained controversial. Ho \& Filippenko (1996) used

velocity dispersion data to estimate a mass of $3.3 \pm 0.5 \times 10^{5} \mathrm{M}_{\odot}$ for cluster A. Sternberg (1998) combined photometric data with a velocity dispersion and concluded that in cluster A there are stars present down to the hydrogen burning limit of $0.1 \mathrm{M}_{\odot}$. Arp \& Sandage (1985) obtained integrated spectra of the clusters and found that they have spectra like those of A supergiants. Prada, Greve, \& McKeith (1994) obtained spectra of the Ca II infrared lines of the clusters and concluded, as had Arp and Sandage, that cluster A is dominated by A and B stars and is 13-20 Myrs old. Cluster B, they concluded, is in a red supergiant phase and is 12 Myrs old. O'Connell et al. (1994) estimated the ages of both clusters to be roughly 15 Myrs from integrated V-I 
colors obtained from HST images. In optical ground-based spectra Delgado et al. (1997) detected the signature of Wolf-Rayet stars in cluster A as well as the signature of young, massive stars in both clusters. They concluded that each cluster had undergone two episodes of star formation separated by about 6 Myrs: 3 and 9 Myrs in cluster A and 2 and 8 Myrs in cluster B. However, De Marchi et al. (1997) from HST images concluded that cluster A is actually two clusters, one that is dominated by red supergiants and one that is younger and contains the Wolf-Rayet stars.

Here we examine some additional data on the structures and ages of these super star clusters. In addition we will include in some of the analysis below two other clusters from Table 2 for comparison. The comparison clusters were chosen to be well-resolved and among the brightest few clusters after clusters $\mathrm{A}$ and B: Cluster 30 has an $\mathrm{M}_{V}$ of -11 and cluster 35 has an $\mathrm{M}_{V}$ of -10 .

\subsection{Structure}

In Figure 6 we show contour plots of clusters A, B, 30, and 35. We can see from Figure 6 that cluster $\mathrm{A}$ has two peaks in its two-dimensional profile, as first pointed out by O'Connell et al. (1994) and later analyzed De Marchi et al. (1997). The peaks, designated A1 and A2 by De Marchi et al., are separated by $0.18^{\prime \prime}$, which is $2.2 \mathrm{pc}$ at the galaxy, and the fainter peak is southeast of the brighter peak. Clusters B, 30, and 35, on the other hand, are relatively round. In the outer parts, cluster B is not entirely symmetrical, being elongated in one dimension, but the deviation is small.

Because of interest in structure within clusters A and B, we have produced two-dimensional ratio maps of these clusters using the $\mathrm{F} 555 \mathrm{~W}$ and $\mathrm{F} 814 \mathrm{~W}$ images. We subtracted local background galaxy from each cluster in each image, and aligned all of the images to the medium F555W exposure. We combined the short, medium, and long exposures, replacing the saturated pixels with values from the unsaturated exposures. The resulting ratio images are shown in Figure 7 . One can see that both clusters are relatively blue in the centers. In cluster A the two luminosity peaks A1 and A2 are very similar in color and both are blue. The reddest regions are located in the outer parts of cluster A and are not obviously associated with any one peak. This is not what one would expect if one component is dominated by red supergiants and the other is not as suggested by De Marchi et al. (1997). However, the large overlap of the two components makes it hard to disentangle the outer parts of the two sub-clusters. Cluster B, on the other hand, is more uniform in appearance, and the center is not as blue although there is a bluer clump of stars to the southeast of the center.

We have also experimented with deconvolving components A1 and A2 in cluster A, as had De Marchi et al. (1997). We used point-spread-function deconvolution (DAOPHOT, Stetson 1987) with various combinations of clusters B, 30, and 35 with Gauss, Lorentz, Penny, and Moffat fitting functions to determine the point-spread-function. We also fit two two-dimensional Gaussians to the peaks. Residuals were fairly high in all cases arguing that clusters B, 30, 35, and 
a two-dimensional Gaussian do not well represent the structures of A1 and A2. Nevertheless, the derived parameters are a rough indication of the characteristics of the two components. We find that component A1 is brighter than A2 by about $1.7 \pm 0.2$ magnitudes in F555W. De Marchi et al. (1997) found a difference of 1.3 magnitudes. Component A1 has an (F555W-F814W) $)_{0}$ color of 0.13. A2 is 0.06 magnitude redder than A1 in these filters, but within the uncertainties one could also say that A1 and A2 have the same colors. De Marchi et al. found A2 to be 0.1 magnitude bluer in $(\mathrm{F} 439 \mathrm{~W}-\mathrm{F} 555 \mathrm{~W})_{0}$. From the two-dimensional Gaussian fits to the F555W image we find that A1 is $2.5 \times 1.9 \mathrm{pc}\left(0.21 \pm 0.01^{\prime \prime} \times 0.155 \pm 0.007^{\prime \prime}\right) \mathrm{FWHM}$ at a position angle of $35^{\circ}$, and A2 is $1.9 \times 1.4 \mathrm{pc}\left(0.15^{\prime \prime} \pm 0.01 \times 0.12 \pm 0.02^{\prime \prime}\right) \mathrm{FWHM}$ at a position angle of $105^{\circ}$. De Marchi et al. measure a half light radius of $0.15^{\prime \prime}$ for A1 and of $0.17^{\prime \prime}$ for A2.

\subsection{Integrated and Surface Brightness Profiles}

The surface brightness and integrated radial profiles of clusters A, B, 30, and 35 are shown in Figure 8. The annuli were measured in steps of 2 pixels which is $0.09^{\prime \prime}$. This choice of step size is small enough with respect to the size of the cluster to give interesting radial information. However, it is still much larger than uncertainties in aligning the images. Uncertainties due to variations in the point-spread-function between filters are expected to be less than $1 \%$ for radii $\geq 4$ pixels, but the inner most point in radial profile plots will be affected especially F555W-F814W (Holtzman et al. 1995a). To increase the signal-to-noise, we found it necessary to integrate over a 4-pixel wide annuli for the annular color profiles.

The fluxes lost due to the saturated pixels in the images of clusters A and B in the medium and long F555W and F814W exposures have been corrected using the short exposures. The final aperture photometry is the average of the photometry in the three exposures, and the uncertainties are the dispersion around the mean. These are observed magnitudes, and F336W has not been corrected for red leak here.

In terms of colors most of the clusters are fairly uniform with radius. All of the clusters are bluer at the center in $\mathrm{F} 555 \mathrm{~W}-\mathrm{F} 814 \mathrm{~W}$ by $0.05-0.1$ magnitude, but this is probably due to the difference in point-spread-function between these two filters (Matthews et al. 1999). The F555W-F814W color then quickly becomes redder, reaching a level that does not change significantly with radius. In F336W-F555W, the behaviour is somewhat different. F336W-F555W remains fairly constant with radius in clusters A and 30. On the other hand, F336W-F555W in clusters B and 35 becomes steadily bluer with radius, by of order 0.1 magnitude.

The half-light radii $\mathrm{R}_{0.5}$ in F555W are given in Table 3. They are 2.2-3.1 pc for clusters A, $\mathrm{B}, 30$, and 35. Our measured values for clusters $\mathrm{A}$ and $\mathrm{B}$ are very close to those measured by O'Connell et al. (1994) from Cycle 1 HST data. A typical value of $\mathrm{R}_{0.5}$ for globular clusters is $3-4$ pc with a range from 1-8 pc (van den Bergh, Morbey, \& Pazder 1991), assuming no dynamical 
evolution of the clusters. Thus, clusters A, B, 30, and 35 have half-light radii that are nearly typical of globular clusters. Thus, clusters A and B have the luminosities (discussed in Section 5) and $R_{0.5}$ of a typical Milky Way globular cluster. In addition, Ho \& Filippenko (1996) have determined the dynamical mass of cluster A to be $3.3 \pm 0.5 \times 10^{5} \mathrm{M}_{\odot}$. An average mass of a globular cluster is $6 \times 10^{5} \mathrm{M}_{\odot}$ with a range in masses of $10^{4} \mathrm{M}_{\odot}$ to $4 \times 10^{6} \mathrm{M}_{\odot}$ (Pryor \& Meylan 1993). Thus, cluster A clearly has many attributes of a young version of a globular cluster.

Clusters 30 and 35 are the reddest clusters in our sample and, with $\mathrm{M}_{V}$ of -11 and -10 , are several magnitudes fainter than A and B. In Figure they lie near the 1 Gyr mark of the $Z=0.004$ cluster evolutionary track. If they are truely that old, then at 10 Myrs they would have been comparable to cluster A in luminosity, implying that super star cluster formation has occurred at various epochs in the past. Because we have assumed a light reddening for these clusters, it is unlikely that we have overcorrected for reddening and that they are consequently really bluer. However, it is possible that the colors of clusters 30 and 35 are dominated by a few red supergiant stars. If clusters 30 and 35 are only 30 Myrs old, as stochastic uncertainties might allow, their $\mathrm{M}_{V}$ at 10 Myrs would have been -12 and -11 , still placing them in the regime of smaller globular clusters and of R136 in the LMC. Spectra would be necessary to distinguish between these possibilities.

We have fit the surface brightness profiles of clusters B, 30, and 35 with a King model (King 1962). Cluster A is excluded from this analysis because it is clearly not spherically symmetric. Following the proceedure outlined by King, we used the inner and outer parts of the profile to determine first guesses of key parameters. We plotted R-squared against the inverse of the flux and fit the inner part of the profile to determine the core radius $r_{c}$ and the central flux $\mathrm{f}_{0}$. We plot $1 / \mathrm{r}$ against the square-root of the flux and fit the outer part of the profile to get an estimate for the terminal radius $\mathrm{r}_{t}$. With these first guesses, we then explored the parameter space of reasonable solutions, determining the best fit and the range of values that are reasonable for these three variables. The uncertainties in the former are determined from the latter. The best fit for each cluster is shown as the solid line in Figure 8. Fit parameters are listed in Table 3.

We have used the list of structural parameters for present-day Milky Way globular clusters given by Djorgovski (1993) and Trager, Djorgovski, \& King (1993) to compile a range and average of the core radius $\mathrm{r}_{c}$ and the concentration index c. We included globular clusters with data quality indices given by the authors of 1 or 2 and excluded clusters identified as having undergone core collapse. This yields an average core radius for 89 globular clusters of $2.6 \pm 3.9$ pc with a total range of $0.2-23$ pc. NGC 1569 's clusters B, 30, and 35, by comparison, have core radii of 0.3-0.8 pc. The core radii of NGC 1569's clusters are smaller than that of the average Milky Way globular cluster, but within the range that is observed. For 95 Milky Way globular clusters the average concentration index is $1.4 \pm 0.4$, with a total range of $0.6-2.4$. The concentration index of the clusters in NGC 1569 are 1.7-2.0. The clusters in NGC 1569 have concentration indices that are within range of what is observed in Milky Way globulars today but larger, that is less concentrated, than the average. 
Thus, cluster A in NGC 1569 has the half-light radius, luminosity, and mass of a fairly normal globular cluster, as seen in the Milky Way today. However, unlike globular clusters seen today, it is not spherically symmetric like the Milky Way globulars or elliptically symmetric like the LMC globulars. However, the globular clusters may have been rounded-out over time by the tidal forces in the Milky Way (Goodwin 1997). Cluster A has two luminosity peaks that could be sub-clusters. Perhaps the two components will merge in the future. Cluster B also has the half-light radius and luminosity of a typical globular cluster. In addition its core radius is at the small end of the range of globulars and it is not as concentrated. Clusters 30 and 35 have half-light radii, core radii, and luminosities that are a bit smaller than the average of the globulars but within the range observed. However, they are not as concentrated overall as the average globular.

\subsection{The Stellar CO Feature}

The near-infrared stellar absorption features of $\mathrm{CO}$ have been found to be useful for placing limits on the age of a coeval stellar population. The features around 2.3 microns are found in stellar spectra beginning with late G stars and becoming stronger with later spectral type of the star (Baldwin et al. 1973). Thus, in a young cluster the CO features are sensitive to the presence of massive stars entering the red supergiant evolutionary phase. Prior to the onset of the red supergiant phase, a young coeval cluster will exhibit no CO features, and then as the red supergiants appear the CO features appear in the integrated cluster spectrum. For the metallicity of NGC 1569, this occurs at 7 Myrs (Mayya 1997, Origlia et al. 1999). While the red supergiant peak only lasts $\sim 5-7$ Myrs (Bica et al. 1990, Origlia et al. 1999), after that other red stars continue to contribute to the CO feature, including an AGB phase at 100 Myrs (Olofsson 1989, Bica et al. 1990). Thus, the CO features can be used to set an upper or lower limit on the age of the clusters depending on whether the features are present or not.

Therefore, we undertook to measure the near-infrared stellar CO absorption features in the super star clusters in NGC 1569. The near-infrared spectra of clusters A and B are shown in Figure 9. The ${ }^{12} \mathrm{CO}(2,0)$ and ${ }^{12} \mathrm{CO}(3,1)$ stellar absorption features around 2.3 microns are prominent. The equivalent widths of these features are given in Table 4 for the clusters and other stars that were observed for comparison. For cluster A this measurement integrates over both sub-clusters (O'Connell et al. 1994, De Marchi et al. 1997). One can see that the equivalent widths of the stellar CO spectral features in clusters A and B are very strong. They are, in fact, comparable to the equivalent widths of those features in the spectra of $\mathrm{K}$ and $\mathrm{M}$ giants and supergiants. Uncertainties in the equivalent widths are not given because the uncertainties are dominated by systematics. From the observation of the F7V star, that should have an equivalent width of zero, we estimate that the uncertainty in the equivalent widths is of order $2 \AA$.

The large $\mathrm{CO}$ equivalent widths in the super star clusters indicate the presence of a significant population of cool, luminous stars, though without further information we cannot determine the exact types. The dilution of the $\mathrm{CO}$ absorption features by the light of warmer stars means that 
the dominant cool types have equivalent widths $>30 \AA$ and are probably red supergiants. Because both clusters show the strong signatures of red supergiants, then, their ages must be $\geq 7$ Myrs. The integrated colors, as shown in Figure 1 suggest that cluster A is at the low end of this limit, while cluster B is perhaps somewhat older.

Prada et al. (1994) observed the Ca II near-infrared spectral features in clusters A and B. They concluded that cluster B is in the red supergiant phase but that cluster A, with weaker Ca II, is older. However, the Ca II calibrations of Bica et al. (1990) do not preclude a younger age for cluster A. As discussed above, the integrated colors of cluster A point to a younger age.

\subsection{Resolved Stars}

Photometry of resolved stars in the outer parts of clusters A and B are shown superposed on color-magnitude diagrams of the entire measured field star population in Figures 10. The stars are shown as a function of distance from the center of the clusters in Figure 11.

As anticipated by O'Connell et al. (1994), a large component of the resolved stars in and near clusters $\mathrm{A}$ and $\mathrm{B}$ are red supergiants. These are stars with $\mathrm{M}_{V} \leq-4$ and $\mathrm{V}-\mathrm{I} \geq 1.5$. In cluster A there are approximately 8 stars with the colors and absolute magnitudes of red supergiants. These are all located along the edge of the included region around the cluster. The rest, and the majority, of the resolved stars are very bright stars in the blue plume extending from about 23.5 to 19 in F555W. This corresponds to -5.2 to -9.7 for an $\mathrm{E}(\mathrm{B}-\mathrm{V})_{t}$ of 0.56 . Early O-type supergiants have $\mathrm{M}_{V}$ of order -7 , and we do see a large clump of blue stars between -5.5 and -7 in cluster $\mathrm{A}$. These are likely massive $\mathrm{O}$ stars. The color-magnitude diagram of cluster A, therefore, is consistent with the interpretation of other data that there are two stellar populations present or a spread in ages (Delgado et al. 1997).

Of the resolved stars in cluster $\mathrm{A}, 16$ have $\mathrm{M}_{V} \leq-7$, even brighter than an $\mathrm{O}$ supergiant (Conti \& Underhill 1988). What are these brightest stars? A possibility is that they are clumps of stars although there would have to be as many as 10 stars in some cases, but a more intriguing, and equally likely, possiblity is that they are evolved massive stars. For example, Luminous Blue Variables (LBVs), which come from stars $\geq 85 \mathrm{M}_{\odot}$ (Massey, Waterhouse, \& DeGioia-Eastwood 2000), can be very bright in V: S Doradus in the LMC is currently -9.7 (Massey 2000). Yet, because they are cooler, LBVs can be brighter than $\mathrm{O}$ supergiants or Wolf-Rayet stars. Furthermore, LBVs have roughly the same ages as Wolf-Rayet stars, which come from stars more massive than about $30 \mathrm{M}_{\odot}$ (Massey, Waterhouse, \& DeGioia-Eastwood 2000), and so their presence is consistent with the presence of 20-40 WN-type stars in the cluster, estimated by Delgado et al. (1997). Cluster A is comparable to a globular cluster. Therefore, it also likely contains millions of stars and, thus, as in R136, we expect there to be a considerable massive star population in the cluster, including numerous of the most massive stars known (Massey \& Hunter 1998). For ages of order $4-5$ Myrs, these very massive stars will not yet have exploded as 
supernovae. The presence of so many Wolf-Rayet stars confirms this picture, and since a large number of Wolf-Rayet stars are present, it would not be a surprise to find LBVs too.

The resolved stellar population in and near cluster B, on the other hand, is markedly different in make-up compared to that in cluster A. There is a clump of bright, blue stars. These are comparable in magnitude to $\mathrm{O}$ supergiants and main sequence stars. The very brightest blue stars seen in cluster A are not present in cluster B. However, the majority of the stars that we have resolved are red supergiants. The large number of red supergiants are consistent with the strong stellar CO feature and the conclusion that cluster B is in its red supergiant phase.

\subsection{Ages}

So, where does this leave us concerning the ages of clusters A and B? For cluster A the integrated colors and the presence of Wolf-Rayet stars argue for ages of order 4-5 Myrs, and the presence of red supergiants and the stellar CO equivalent width argues for an age greater than 7 Myrs. This apparent contradiction between a young age and an older age is the reason that De Marchi et al. (1997) suggested that one of the sub-clusters in A is the young object and the other is the older object. However, with this scenario one might expect some spatial separation in stellar populations and hence colors. While plausible, our two-dimensional color map does not offer support of this. Alternatively, cluster A may have formed over an extended period of time, as suggested by Delgado et al. (1997), which would potentially allow the older and younger stars to be more spatially mixed. An age spread of only a few Myrs in the formation of the massive stars is all that would be required. Furthermore, Massey (1998) and Massey \& Johnson (1998) have shown that RSG and Wolf-Rayet stars are often found together in OB associations. The same mass range of massive stars go through the RSG and the Wolf-Rayet phases, and this is especially true for a metal poor stellar population. Thus, RSGs and Wolf-Rayet stars can coexist even in a coeval population.

The data for cluster B, on the other hand, are more consistent. The colors suggest 10-30 Myrs, with uncertainty because the cluster does not fall near the cluster evolutionary track, the large number of red supergiants and the $\mathrm{CO}$ equivalent width argues for an age greater than 7 Myrs, and the Ca II feature also suggests that the cluster is in the red supergiant phase (Prada et al. 1994). Thus, it appears that cluster B is of order 10-20 Myrs old and, therefore, older than the young component of cluster $\mathrm{A}$, but roughly comparable in age to the older component of $\mathrm{A}$.

According to Vallenari \& Bomans (1996) and Greggio et al. (1998), the bulk of the current starburst in NGC 1569 ended 5-10 Myrs ago and extended back about 100 Myrs from that. It is expected that super star clusters might form during such an episode, although it is surprising that cluster formation was so strongly concentrated to the end of that period. Once clusters form, they are expected to disperse the gas relatively quickly (as seen in Figure 2). According to Israel \& van Driel (1990), the size of the HI hole - diameter 200 pc-around the clusters is consistent 
with an age of 2-10 Myrs for reasonable input parameters to stellar wind models. The hole in the $\mathrm{H} \alpha$, seen in Figure 2, is 170 pc in diameter and is also consistent with this picture. In spite of the obvious disruption of the ISM in the center of the galaxy, star formation is, nevertheless, continuing: Taylor et al. (1999) have mapped 5 giant molecular clouds just outside the HI hole to the northwest of the two super star clusters.

\section{Summary}

We have examined star clusters in HST images of the irregular, starburst galaxy NGC 1569 . In addition to the super star clusters $\mathrm{A}$ and $\mathrm{B}$ that are well known, we identified 45 other clusters that are compact but resolved. We also include one known and easily isolated OB association in our study. The OB association is the youngest of the clusters.

Integrated colors of the clusters span a large range. Comparison with coeval evolutionary models at $Z=0.004$ suggest that the ages range from a few Myrs to nearly 1 Gyr, but a better fit to some cluster colors may be obtained with more metal-rich $Z=0.008$ models. Uncertainties due to stochastic effects, reddening, background subtraction, and modelling make it difficult to pin ages of clusters down from integrated photometry. However, a large number of the clusters have colors consistent with ages of 4-6 Myrs and most with ages $<30$ Myrs. Thus, most of these clusters have been formed at the tail end of the recent burst of star formation and were not formed continuously during the starburst.

We examined the surface brightness profiles of the super star clusters A and B and two other very bright clusters. We fit King model profiles to all but cluster A. The clusters have half-light radii and core radii that are in the range observed for present-day globular clusters in our Galaxy. However, the core radii of the NGC 1569 clusters are on the small end of the range and they are somewhat less concentrated that the average globular. Clusters A and B have luminosities, and cluster A has a mass, that place them as young versions of typical globular clusters. The other two clusters, and likely several others in the sample as well, are similar to a small globular cluster and to R136 in the LMC. Thus, whatever conditions facilitated the recent starburst were also adequate for producing numerous compact clusters that are comparable to small globular or populous clusters.

Cluster B and two other clusters chosen for comparison are nearly spherically symmetric. However, cluster A, as was known from previous work, has two peaks in its light distribution. This has been interpretted by others as evidence for the presence of two sub-clusters. The colors of the clusters are approximately constant with radius, with $\mathrm{F} 336 \mathrm{~W}-\mathrm{F} 555 \mathrm{~W}$ becoming bluer by 0.1 magnitude in two of the clusters.

We have examined resolved stars in the outer parts of clusters A and B. We find that cluster A contains many bright blue stars. Some of the blue stars are bright enough to be evolved massive

stars such as LBVs. The presence of such stars is consistent with the presence of a large number of 
Wolf-Rayet stars and the expected large number of very massive stars for the mass of the cluster. The resolved star population around cluster A also contains a small population of red supergiants. The presence of both blue and red massive star populations are consistent with the view that the two sub-clusters have different ages, but our two-dimensional color map of cluster A suggests that the two luminosity peaks are comparably blue and that the reddest stars, located in the outer parts of the cluster, are not obviously preferentially associated with one of the peaks. Instead, the presence of both RSG and Wolf-Rayet stars in a metal poor coeval population is not inconsistent with the evolution of massive stars, or alternatively there is a small age spread of several Myrs within the cluster. The stars that we resolved around cluster B, on the other hand, contain a small population of more normal blue massive stars and a large population of red supergiants. The presence of the red supergiants is consistent with the view that cluster B is in its red supergiant phase.

We have measured the near-infrared stellar CO feature in clusters $\mathrm{A}$ and $\mathrm{B}$ and find that strong absorption features are present in both. This indicates that red supergiants are present, as is confirmed by photometry of the resolved stars in the outer parts of the clusters. The implied ages are $\geq 7$ Myrs. Other age indicators are consistent with a picture in which cluster $\mathrm{B}$ is of order 10-20 Myrs old. It is likely that the clusters have contributed to clearing a hole in the gas, seen in $\mathrm{HI}$ and $\mathrm{H} \alpha$. The $\mathrm{H} \alpha$ hole is $170 \mathrm{pc}$ in diameter, and the timescale to form it is comparable to the age of cluster $\mathrm{B}$.

We wish to thank Allan Watson for help with the near-infrared observations. Support for this work was provided by NASA through grant number GO-06423.01-95A to D.A.H. and grant number NAG5-6403 to R.W.O. 
Table 1. The HST Observations

\begin{tabular}{lrr}
\hline \hline Filter & Exposure time (s) & Image name \\
\hline F336W & 400 & U54G0107 \\
F336W & 400 & U54G0108 \\
F555W & 50 & U54G0103 \\
F555W & 140 & U54G0104 \\
F555W & 140 & U54G0105 \\
F555W & 300 & U54G010C \\
F555W & 300 & U54G010D \\
F814W & 50 & U54G0106 \\
F814W & 100 & U54G0109 \\
F814W & 100 & U54G010a \\
F814W & 300 & U54G010b \\
F656N & 800 & U54G0101 \\
F656N & 800 & U54G0102 \\
\hline
\end{tabular}


Table 3. Cluster Parameters

\begin{tabular}{lccccccc}
\hline \hline Cluster & $\begin{array}{c}\mu_{0} \\
(\mathrm{mag})\end{array}$ & $\begin{array}{c}\mathrm{r}_{t} \\
\left({ }^{\prime \prime}\right)\end{array}$ & $\begin{array}{c}\mathrm{r}_{c} \\
\left({ }^{\prime \prime}\right)\end{array}$ & $\begin{array}{c}\mathrm{r}_{c} \\
(\mathrm{pc})\end{array}$ & $\mathrm{c}^{\mathrm{a}}$ & $\begin{array}{c}\mathrm{R}_{0.5} \mathrm{~b} \\
\left({ }^{\prime \prime}\right)\end{array}$ & $\begin{array}{c}\mathrm{R}_{0.5} \\
(\mathrm{pc})\end{array}$ \\
\hline $\mathrm{A}$ & $\ldots$ & $\ldots$ & $\ldots$ & $\ldots$ & $\ldots$ & 0.19 & 2.3 \\
$\mathrm{~B}$ & $12.48 \pm 0.12$ & $4.78 \pm 2.0$ & $0.048 \pm 0.009$ & $0.58 \pm 0.11$ & $2.0 \pm 0.3$ & 0.26 & 3.1 \\
30 & $13.5 \pm 0.5$ & $1.25 \pm 0.75$ & $0.026 \pm 0.005$ & $0.32 \pm 0.06$ & $1.7 \pm 0.3$ & 0.20 & 2.5 \\
35 & $15.5 \pm 0.25$ & $4.0 \pm 2.0$ & $0.062 \pm 0.006$ & $0.75 \pm 0.07$ & $1.8 \pm 0.2$ & 0.18 & 2.2 \\
Globular cluster & $\ldots$ & $\ldots$ & $\ldots$ & $2.6 \pm 3.9$ & $1.4 \pm 0.4$ & $\ldots$ & 3 \\
\hline
\end{tabular}

${ }^{\mathrm{a}}$ Concentration index $\log \left(\mathrm{r}_{t} / \mathrm{r}_{c}\right)$.

${ }^{\mathrm{b}}$ Half-light radius in $\mathrm{F} 555 \mathrm{~W}$.

${ }^{\mathrm{c}}$ Average parameters of Milky Way globular clusters as seen today. From Djorgovski (1993); Pryor \& Meylan (1993); and Trager, Djorgovski, \& King (1993). 
Table 4. Stellar CO Equivalent Widths

\begin{tabular}{lccc}
\hline \hline Object & Spectral Type & $\begin{array}{c}{ }^{12} \mathrm{CO}(2,0) \\
(\AA)\end{array}$ & $\begin{array}{c}{ }^{12} \mathrm{CO}(3,1) \\
(\AA)\end{array}$ \\
\hline Cluster A & & 25.4 & 19.7 \\
Cluster B & & 26.4 & 18.8 \\
HR8837 & A0V & 0 & 0 \\
HR1040 & A0Ia & 0 & 0 \\
$\sigma$ And & A2Va & 0 & 0 \\
64 Psc & F7V & 0 & 2.3 \\
HR8752 & G5Ia & 0 & 0 \\
$\xi$ And & KOIII & 9.3 & 7.6 \\
$35 \sigma P e r$ & K3III & 21.2 & 18.0 \\
HR8726 & K5Ib & 33.7 & 22.3 \\
$\chi$ Peg & M2III & 26.6 & 17.7 \\
$\mu$ Cep & M2Ia & 40.6 & 26.5 \\
\hline
\end{tabular}




\section{REFERENCES}

Ables, H. D. 1968, PhD Thesis, University of Texas

Arp, H., \& Sandage, A. 1985, AJ, 90, 1163

Baldwin, J. R., Frogel, J. A., \& Persson, S. E. 1973, ApJ, 184, 427

Barth, A. J., Ho, L. C., Filippenko, A. V., \& Sargent, W. L. 1995, AJ, 110, 1009

Bica, E., Alloin, D., Santos, J. F. C., Jr. 1990, A\&A, 235, 103

Bouchet, P., Lequeux, J., Maurice, E., Prevot, L., Prevot-Burnichon, M. L. 1985, A\&A, 149, 330

Brocato, E., Castellani, V., Raimondo, G., \& Romaniello, M. 1999, A\&AS, 136, 65

Burstein, D., \& Heiles, C. 1984, ApJS, 54, 33

Cardelli, J. A., Clayton, G. C., \& Mathis, J. S. 1989, ApJ, 345, 245

Conti, P. S., \& Underhill, A. B. 1988, O Stars and Wolf-Rayet Stars, NASA SP-497 (Washington, D.C., NASA)

Conti, P., \& Vacca, W. 1994, ApJ, 423, L97

De Marchi, G., Clampin, M., Greggio, L., Leitherer, C., Nota, A., \& Tosi, M. 1997, ApJ, 479, L27

de Vaucouleurs, G., de Vaucouleurs, A., \& Pence, W. 1974, ApJ, 194, L119

Della Ceca, R., Griffiths, R. E., Heckman, T. M., \& Mackenty, J. W. 1996, ApJ, 469, 662

Devost, D., Roy, J.-R., \& Drissen, L. 1997, ApJ, 482, 765

Djorgovski, S. 1993, in Structure and Dynamics of Globular Clusters, ASP Conference Series Vol. 50, eds. S. G. Djorgovski and G. Meylan (San Francisco:ASP), p 373

Dolphin, A. E., \& Hunter, D. A. 1998, AJ, 116, 1275

Drissen, L., \& Roy, J.-R. 1994, PASP, 106, 974

Gallagher, J. S., Hunter, D. A., \& Tutukov, A. 1984, ApJ, 284, 544

Gallagher, J. S., \& Smith, L. J. 1999, MNRAS, 304, 540

Girardi, L., \& Bica, E. 1993, A\&A, 274, 279

González Delgado, R. M., Leitherer, C., Heckman, T., Ceviño, M. 1997, ApJ, 483, 705

Goodwin, S. P. 1997, MNRAS, 286, L39

Greggio, L., Monica, T., Clampin, M., De Marchi, G., Leitherer, C., Nota, A., \& Sirianni, M. 1998, ApJ, 504, 725

Harris, W. E. 1991, ARA\&A, 29, 543

Heckman, T. M., Dahlem, M., Lehnert, M. D., Fabbiano, G., Gilmore, D., \& Waller, W. H. 1995, ApJ, 448, 98

Ho, L. C., \& Filippenko, A. V. 1996, ApJ, 466, L83 
Holtzman, J., Hester, J. J., Casertano, S., Trauger, J. T., Watson, A. M., Ballester, G. E., Burrows, C. J., Clarke, J. T., Crisp, D., Evans, R. W., Gallagher, J. S., III, Griffiths, R. E., Hoessel, J. G., Matthews, L. D., Mould, J. R., Scowen, P. A., Stapelfeldt, K. R., \& Westphal, J. A. 1995a, PASP, 107, 156

Holtzman, J. A., Burrows, C. J., Casertano, S., Hester, J. J., Trauger, J. T., Watson, A. M., \& Worthey, G. 1995b, PASP, 107, 1065

Holtzman, J. A., Faber, S. M., Shaya, E. J., Lauer, T. R., Groth, J., Hunter D. A., Baum, W. A., Ewald, S. P., Hester, J. F., Light, R. M., Lynds, C. R., O’Neil, E. J., Jr., \& Westphal, J. A. 1992, AJ, 103, 691

Hunter, D. A. 1995, Rev. Mexicana de A\&A (Serie de Conferencias), 3, 1

Hunter, D. A., \& Gallagher, J. S. 1997, ApJ, 475, 65

Hunter, D. A., Hawley, W. N., \& Gallagher, J. S. 1993, AJ, 106, 1797

Hunter, D. A., Light, R. M., Holtzman, J. A., Lynds, R., O’Neil, E. J., Jr., \& Grillmair, C. J. 1997, ApJ, 478, 124

Hunter, D. A., O’ Connell, R. W., \& Gallagher, J. S. 1994, AJ, 108, 84

Hunter, D. A., Shaya, E. J., Holtzman, J. A., Light, R. M., O’Neil, E. J., Jr., \& Lynds, R. 1995, ApJ, 448, 179

Israel, F. P. 1988, A\&A, 194, 24

Israel, F. P., \& van Driel, W. 1990, A\&A, 236, 323

King, I. 1962, AJ, 67, 471

Kobulnicky, H. A., \& Skillman, E. D. 1997, ApJ, 489, 636

Leitherer, C., Schaerer, D., Goldader, J. D., González Delgado, R. M., Robert, C., Kune, D. F., de Mello, D. F., Devost, D., \& Heckman, T. M. ApJS, 123, 3

Massey, P. 1998, ApJ, 501, 153

Massey, P. 2000, PASP, in press

Massey, P., \& Hunter, D. A. 1998, ApJ, 493, 180

Massey, P., \& Johnson, O. 1998, ApJ, 505, 793

Massey, P., Waterhouse, E., \& DeGioia-Eastwood, K. 2000, AJ, in press

Matthews, L. D., Gallagher, J. S., III, Krist, J. E., Watson, A. M., Burrows, C. J., Griffiths, R. E., Hester, J. J., Trauger, J. T., Ballester, G. E., Clarke, J. T., Crisp, D., Evans, R. W., Hoessel, J. G., Holtzman, J. A.; Mould, J. R., Scowen, P. A., Stapelfeldt, K. R., Westphal, J. A. 1999, AJ, 118, 208

Mayya, Y. D. 1997, ApJ, 482, L149

O’Connell, R. W., Gallagher, J. S., \& Hunter, D. A. 1994, ApJ, 433, 65 
O’Connell, R. W., Gallagher, J. S., Hunter, D. A., \& Colley, W. 1995, ApJ, 446, L1

Olofsson, K. 1989, A\&AS, 80, 317

Origlia, L., Goldader, J. D., Leitherer, C., Schaerer, D., \& Oliva, E. 1999, ApJ, 514, 96

Prada, F., Greve, A., \& McKeith, C. D. 1994, A\&A, 288, 396

Pryor, C., \& Meylan, G. 1993, in Structure and Dynamics of Globular Clusters, ASP Conference Series Vol. 50, eds. S. G. Djorgovski and G. Meylan (San Francisco:ASP), p 357

Salpeter, E. 1955, ApJ, 121, 161

Santos, J. F. C., Jr., \& Frogel, J. A. 1997, ApJ, 479, 764

Sternberg, A. 1998, ApJ, 506, 721

Stetson, P. B. 1987, PASP, 99, 191

Stil, J. M., \& Israel, F. P. 1998, A\&A, 337, 64

Swaters, R. 1999, Ph.D. thesis, Rijksuniversiteit Groningen

Taylor, C. L., Hüttemeister, S., Klein, U., \& Greve, A. 1999, A\&A, 349, 424

Tomita, A., Ohta, K., \& Sait_o, M. 1994, PASJ, 46, 335

Trager, S. C., Djorgovski, S., \& King, I. R. 1993, in Structure and Dynamics of Globular Clusters, ASP Conference Series Vol. 50, eds. S. G. Djorgovski and G. Meylan (San Francisco:ASP), p 347

Vallenari, A., \& Bomans, D. J. 1996, A\&A, 313, 713

van den Bergh, S., Morbey, C., \& Pazder, J. 1991, ApJ, 375, 594

Walborn, N. R., \& Blades, J. C. 1997, ApJS, 112, 457

Waller, W. H. 1991, ApJ, 370, 144

Whitmore, B. C., Schweizer, F., Leitherer, C., Borne, K., \& Carmelle, R. 1993, AJ, 106, 1354

This preprint was prepared with the AAS $\mathrm{LAT}_{\mathrm{E}} \mathrm{X}$ macros v4.0. 
Fig. 1.- Mosaic of the HST CCD images of the 140 second F555W exposure of NGC 1569.

Fig. 2.- False-color display of the PC image of the center of NGC 1569 shows the two super star clusters and the surrounding gas. The medium exposure F555W is shown as blue, the medium exposure $\mathrm{F} 814 \mathrm{~W}$ is shown as green, and the $\mathrm{H} \alpha$ image is displayed in red. The orientation is shown in Figure 1, and the scale is shown in Figure 3(a).

Fig. 3. - Star clusters discussed in the text are identified on the WFPC2 images: a) PC1, b) WF2, and c) WF4. The circle size is the size of the aperture used in the integrated photometry of the clusters.

Fig. 4.- Star clusters are shown in a UVI color-color diagram. Three clusters with $(\mathrm{U}-\mathrm{V})_{0}>0.8$ and high uncertainties in that color are not included in the plot. The solid curve in the upper panel is an evolutionary track for a cluster with instantaneous star formation and a metallicity of 0.004 and a Salpeter (1955) stellar initial mass function with an upper limit of $100 \mathrm{M}_{\odot}$ (Leitherer et al. 1999). The solid curve in the lower panel are their models for a metallicity of 0.008. Ages 1-9 Myrs in steps of 1 Myrs are marked with x's along these lines; ages 10, 20, and 30 Myrs are marked with open circles. The evolutionary tracks end at $1 \mathrm{Gyr}$. The arrow in the lower left corner of the upper panel is a reddening line for a change of 0.2 in $\mathrm{E}(\mathrm{B}-\mathrm{V})_{t}$. It represents the average of an $\mathrm{O} 6$ and a K5 type spectrum with $\mathrm{A}_{V} / \mathrm{E}(\mathrm{B}-\mathrm{V})=3.1$ and a Cardelli et al. (1989) reddening curve. Clusters $\mathrm{A}$, $\mathrm{B}, 30$, and 35 are plotted as their names rather than as points; their uncertainties are comparable to or smaller than the symbols.

Fig. 5.- Star clusters shown in UVI color-magnitude diagrams. The solid curve is an evolutionary track for a cluster with instantaneous star formation and a metallicity of 0.004 and a Salpeter (1955) stellar initial mass function with an upper limit of $100 \mathrm{M}_{\odot}$ (Leitherer et al. 1999); the dashed line are their models for a metallicity of 0.008. Ages 1-9 Myrs in steps of 1 Myrs are marked with x's along these lines; ages 10, 20, and 30 Myrs are marked with open circles. The evolutionary tracks end at 1 Gyr. The evolutionary tracks have been scaled to the expected mass of cluster A; for other masses the lines would slide up and down in the diagrams. Clusters A, B, 30, and 35 are plotted as their names rather than as points; their uncertainties are comparable to or smaller than the symbols.

Fig. 6.- Contour plots of clusters A and B and two comparison clusters from the 140 second F555W image. The tics for clusters A and B mark every 10 pixels which is $0.455^{\prime \prime}$. The tics for clusters 30 and 35 mark every pixel which is $0.0455^{\prime \prime}$.

Fig. 7.- Color ratio image of (a) cluster A and (b) cluster B. We have divided the F555W images by the F $814 \mathrm{~W}$ images, and averaged the short, medium, and long exposure images. Saturated pixels in the longer exposures have been replaced with values from the shorter exposures. Two contours from the medium exposure $\mathrm{F} 555 \mathrm{~W}$ image are shown superposed in order to outline the cluster and delineate the luminosity peaks. 
Fig. 8.- Integrated and surface brightness profiles in F555W and integrated and annuli color profiles of F555W-F814W and F336W-F555W: a) cluster A, b) cluster B, c) cluster 30, and d) cluster 35 . The solid curves in the surface brightness plots are the best fit King model. To facilitate comparison between the clusters, for each cluster we show the same range in radius, a 0.3 magnitude range in each of the integrated colors, and a 0.5 magnitude range in annuli colors. Uncertainties are shown for all measurements, but in many cases the error bars are smaller than the point size. Magnitudes are not corrected for reddening or red leak.

Fig. 9. - Near-infrared spectra of clusters A and B. The ${ }^{12} \mathrm{CO}(2,0)$ at $22935 \AA$ and ${ }^{12} \mathrm{CO}(3,1)$ at $23227 \AA$ stellar absorption features are prominent.

Fig. 10.- Color-magnitude diagram of resolved stars within a 30 pixel, 1.36" , radius of the cluster center: a) Cluster A and b) Cluster B. The cluster stars in or near the clusters are shown as large starred points. The small x's are the entire resolved stellar population that we measured in NGC 1569. The absolute magnitude shown along the right vertical axis is for a distance of $2.5 \mathrm{Mpc}$ and an $\mathrm{E}(\mathrm{B}-\mathrm{V})_{t}$ of 0.56 and the average responses of $\mathrm{O} 6$ and $\mathrm{K} 5$ spectral energy distributions.

Fig. 11.- Colors and distance from the cluster center of resolved stars within a 30 pixel, 1.36", radius of the cluster centers for the super star clusters a) A and b) B. Colors are not corrected for reddening. 
This figure "fig1.jpeg" is available in "jpeg" format from: http://arxiv.org/ps/astro-ph/0009280v1 
This figure "fig2.jpeg" is available in "jpeg" format from: http://arxiv.org/ps/astro-ph/0009280v1 
This figure "fig3a.jpeg" is available in "jpeg" format from: http://arxiv.org/ps/astro-ph/0009280v1 
This figure "fig3b.jpeg" is available in "jpeg" format from: http://arxiv.org/ps/astro-ph/0009280v1 
This figure "fig3c.jpeg" is available in "jpeg" format from: http://arxiv.org/ps/astro-ph/0009280v1 
This figure "fig4.jpeg" is available in "jpeg" format from: http://arxiv.org/ps/astro-ph/0009280v1 
This figure "fig5.jpeg" is available in "jpeg" format from: http://arxiv.org/ps/astro-ph/0009280v1 
This figure "fig6.jpeg" is available in "jpeg" format from: http://arxiv.org/ps/astro-ph/0009280v1 
This figure "fig7a.jpeg" is available in "jpeg" format from: http://arxiv.org/ps/astro-ph/0009280v1 
This figure "fig7b.jpeg" is available in "jpeg" format from: http://arxiv.org/ps/astro-ph/0009280v1 
This figure "fig8a.jpeg" is available in "jpeg" format from: http://arxiv.org/ps/astro-ph/0009280v1 
This figure "fig8b.jpeg" is available in "jpeg" format from: http://arxiv.org/ps/astro-ph/0009280v1 
This figure "fig8c.jpeg" is available in "jpeg" format from: http://arxiv.org/ps/astro-ph/0009280v1 
This figure "fig8d.jpeg" is available in "jpeg" format from: http://arxiv.org/ps/astro-ph/0009280v1 
This figure "fig9.jpeg" is available in "jpeg" format from: http://arxiv.org/ps/astro-ph/0009280v1 
TABLE 2

Integrated Cluster Photometry ${ }^{\mathrm{a}}$

\begin{tabular}{|c|c|c|c|c|c|c|c|c|c|c|}
\hline Cluster & RA (2000) & DEC (2000) & $\begin{array}{c}\text { Radius } \\
\left({ }^{\prime \prime}\right)\end{array}$ & $\mathrm{E}(\mathrm{B}-\mathrm{V})_{t}$ & $\mathrm{M}_{F 555 W}$ & $(\mathrm{~F} 336 \mathrm{~W}-\mathrm{F} 555 \mathrm{~W})_{0}$ & $(\mathrm{~F} 555 \mathrm{~W}-\mathrm{F} 814 \mathrm{~W})_{0}$ & $\mathrm{M}_{V}$ & $(\mathrm{U}-\mathrm{V})_{0}$ & $(\mathrm{~V}-\mathrm{I})_{0}$ \\
\hline \multirow[t]{2}{*}{$\mathrm{A}$} & $4: 30: 48.19$ & $64: 50: 58.6$ & 1.14 & 0.56 & -13.96 & -1.43 & 0.09 & -13.97 & -0.96 & 0.09 \\
\hline & & & & & 0.00 & 0.00 & 0.00 & 0.00 & 0.00 & 0.00 \\
\hline \multirow[t]{2}{*}{ B } & $4: 30: 48.99$ & $64: 50: 52.7$ & 1.34 & 0.56 & -13.02 & -1.00 & 0.68 & -13.05 & -0.72 & 0.70 \\
\hline & & & & & 0.00 & 0.01 & 0.00 & 0.00 & 0.01 & 0.00 \\
\hline \multirow[t]{2}{*}{3} & $4: 30: 46.31$ & $64: 51: 08.8$ & 0.46 & 0.56 & -9.64 & -0.85 & 0.32 & -9.65 & -0.51 & 0.33 \\
\hline & & & & & 0.01 & 0.05 & 0.01 & 0.01 & 0.05 & 0.01 \\
\hline \multirow[t]{2}{*}{4} & $4: 30: 46.45$ & $64: 51: 00.4$ & 0.23 & 0.63 & -8.66 & -1.53 & -.12 & -8.65 & -0.98 & -0.11 \\
\hline & & & & & 0.01 & 0.05 & 0.03 & 0.01 & 0.05 & 0.03 \\
\hline \multirow[t]{2}{*}{5} & $4: 30: 46.67$ & $64: 50: 54.4$ & 0.46 & 0.56 & -8.55 & -0.61 & 0.48 & -8.56 & -0.33 & 0.49 \\
\hline & & & & & 0.01 & 0.12 & 0.02 & 0.01 & 0.12 & 0.02 \\
\hline \multirow[t]{2}{*}{6} & $4: 30: 46.89$ & $64: 51: 00.6$ & 0.34 & 0.71 & -9.65 & -1.65 & 0.35 & -9.67 & -1.24 & 0.36 \\
\hline & & & & & 0.02 & 0.04 & 0.03 & 0.02 & 0.04 & 0.03 \\
\hline \multirow[t]{2}{*}{7} & $4: 30: 46.96$ & $64: 50: 59.5$ & 0.34 & 0.63 & -9.17 & -0.09 & 0.27 & -9.18 & 0.19 & 0.28 \\
\hline & & & & & 0.02 & 0.12 & 0.03 & 0.02 & 0.12 & 0.03 \\
\hline \multirow[t]{2}{*}{8} & $4: 30: 47.04$ & $64: 51: 06.6$ & 0.23 & 0.63 & -8.59 & -0.82 & 0.75 & -8.62 & -0.56 & 0.77 \\
\hline & & & & & 0.01 & 0.06 & 0.01 & 0.01 & 0.06 & 0.01 \\
\hline 9 & $4: 30: 47.25$ & $64: 51: 03.0$ & 0.31 & 0.63 & -9.12 & -0.67 & 0.84 & -9.15 & -0.43 & 0.85 \\
\hline & & & & & 0.03 & 0.07 & 0.04 & 0.03 & 0.07 & 0.04 \\
\hline 10 & $4: 30: 47.26$ & $64: 51: 02.3$ & 0.71 & 0.63 & -11.91 & -1.15 & 0.45 & -11.93 & -0.81 & 0.46 \\
\hline & & & & & 0.00 & 0.01 & 0.01 & 0.00 & 0.01 & 0.01 \\
\hline 11 & $4: 30: 47.35$ & $64: 50: 48.1$ & 0.46 & 0.56 & -8.32 & 0.12 & 0.66 & -8.34 & 0.30 & 0.67 \\
\hline & & & & & 0.01 & 0.27 & 0.02 & 0.01 & 0.27 & 0.02 \\
\hline 12 & $4: 30: 47.48$ & $64: 50: 57.8$ & 0.23 & 0.56 & -8.39 & -0.61 & 0.20 & -8.40 & -0.26 & 0.21 \\
\hline & & & & & 0.02 & 0.08 & 0.02 & 0.02 & 0.08 & 0.02 \\
\hline 13 & $4: 30: 47.52$ & $64: 50: 58.4$ & 0.27 & 0.56 & -9.64 & -1.48 & -.22 & -9.62 & -0.87 & -0.22 \\
\hline & & & & & 0.01 & 0.03 & 0.02 & 0.01 & 0.03 & 0.02 \\
\hline 14 & $4: 30: 47.69$ & $64: 50: 59.3$ & 0.27 & 0.56 & -8.80 & -1.13 & 0.16 & -8.80 & -0.72 & 0.17 \\
\hline & & & & & 0.01 & 0.05 & 0.03 & 0.01 & 0.05 & 0.03 \\
\hline 15 & $4: 30: 47.83$ & $64: 51: 00.7$ & 0.46 & 0.56 & -10.76 & -0.96 & 0.29 & -10.77 & -0.60 & 0.30 \\
\hline & & & & & 0.01 & 0.02 & 0.01 & 0.01 & 0.02 & 0.01 \\
\hline 16 & $4: 30: 47.89$ & $64: 51: 00.0$ & 0.14 & 0.56 & -7.82 & -1.34 & 0.62 & -7.84 & -1.02 & 0.63 \\
\hline & & & & & 0.03 & 0.06 & 0.03 & 0.03 & 0.06 & 0.03 \\
\hline 17 & $4: 30: 48.11$ & $64: 51: 05.0$ & 0.46 & 0.56 & -8.27 & -0.08 & 0.43 & -8.28 & 0.16 & 0.44 \\
\hline & & & & & 0.03 & 0.27 & 0.04 & 0.03 & 0.27 & 0.04 \\
\hline 18 & $4: 30: 48.07$ & $64: 50: 57.3$ & 0.18 & 0.56 & -7.75 & -0.58 & 0.55 & -7.77 & -0.31 & 0.56 \\
\hline & & & & & 0.04 & 0.15 & 0.05 & 0.04 & 0.15 & 0.05 \\
\hline 19 & $4: 30: 48.11$ & $64: 50: 59.8$ & 0.27 & 0.56 & -8.67 & -1.25 & 0.73 & -8.69 & -0.95 & 0.74 \\
\hline & & & & & 0.03 & 0.07 & 0.04 & 0.03 & 0.07 & 0.04 \\
\hline 20 & $4: 30: 48.20$ & $64: 51: 00.0$ & 0.14 & 0.56 & -9.66 & 1.84 & 0.77 & -9.68 & 1.85 & 0.78 \\
\hline & & & & & 0.01 & 0.24 & 0.01 & 0.01 & 0.24 & 0.01 \\
\hline 21 & $4: 30: 48.24$ & $64: 51: 00.0$ & 0.14 & 0.56 & -8.50 & -1.53 & -.07 & -8.49 & -0.99 & -0.07 \\
\hline & & & & & 0.02 & 0.04 & 0.03 & 0.02 & 0.04 & 0.03 \\
\hline 22 & $4: 30: 48.26$ & $64: 51: 00.4$ & 0.14 & 0.56 & -7.88 & -1.30 & -.16 & -7.87 & -0.74 & -0.15 \\
\hline & & & & & 0.02 & 0.07 & 0.04 & 0.02 & 0.07 & 0.04 \\
\hline 23 & $4: 30: 48.33$ & $64: 51: 00.7$ & 0.23 & 0.56 & -8.16 & -1.22 & 0.03 & -8.16 & -0.75 & 0.04 \\
\hline & & & & & 0.04 & 0.10 & 0.06 & 0.04 & 0.10 & 0.06 \\
\hline 24 & $4: 30: 48.32$ & $64: 51: 01.0$ & 0.02 & 0.56 & -7.82 & -1.09 & 0.88 & -7.84 & -0.82 & 0.89 \\
\hline & & & & & 0.04 & 0.08 & 0.04 & 0.04 & 0.08 & 0.04 \\
\hline 25 & $4: 30: 48.31$ & $64: 50: 57.6$ & 0.18 & 0.56 & -7.77 & -1.35 & 0.10 & -7.78 & -0.89 & 0.11 \\
\hline & & & & & 0.06 & 0.11 & 0.08 & 0.06 & 0.11 & 0.08 \\
\hline 26 & $4: 30: 48.37$ & $64: 50: 58.4$ & 0.27 & 0.56 & -9.85 & -1.13 & 0.36 & -9.86 & -0.77 & 0.37 \\
\hline & & & & & 0.01 & 0.04 & 0.02 & 0.01 & 0.04 & 0.02 \\
\hline 27 & $4: 30: 48.46$ & $64: 51: 00.3$ & 0.14 & 0.56 & -7.99 & -1.41 & -.23 & -7.98 & -0.81 & -0.22 \\
\hline & & & & & 0.01 & 0.04 & 0.03 & 0.01 & 0.04 & 0.03 \\
\hline 28 & $4: 30: 48.48$ & $64: 50: 53.1$ & 0.34 & 0.56 & -9.84 & -0.81 & 0.32 & -9.85 & -0.48 & 0.33 \\
\hline & & & & & 0.01 & 0.03 & 0.01 & 0.01 & 0.03 & 0.01 \\
\hline 29 & $4: 30: 48.60$ & $64: 50: 58.7$ & 0.34 & 0.56 & -9.52 & -0.63 & 0.90 & -9.54 & -0.40 & 0.91 \\
\hline & & & & & 0.01 & 0.04 & 0.02 & 0.01 & 0.04 & 0.02 \\
\hline 30 & $4: 30: 49.22$ & $64: 51: 02.0$ & 0.83 & 0.56 & -11.13 & 0.54 & 0.59 & -11.15 & 0.68 & 0.61 \\
\hline & & & & & 0.00 & 0.05 & 0.01 & 0.00 & 0.05 & 0.01 \\
\hline 31 & $4: 30: 49.37$ & $64: 50: 52.3$ & 0.23 & 0.56 & -8.23 & -1.19 & 0.43 & -8.25 & -0.85 & 0.44 \\
\hline & & & & & 0.02 & 0.08 & 0.03 & 0.02 & 0.08 & 0.03 \\
\hline 32 & $4: 30: 49.57$ & $64: 50: 53.5$ & 0.23 & 0.56 & -8.79 & -1.43 & -.19 & -8.78 & -0.84 & -0.19 \\
\hline & & & & & 0.01 & 0.03 & 0.02 & 0.01 & 0.03 & 0.02 \\
\hline 33 & $4: 30: 49.93$ & $64: 51: 06.3$ & 0.46 & 0.56 & -8.17 & -0.56 & 1.10 & -8.19 & -0.33 & 1.12 \\
\hline & & & & & 0.02 & 0.16 & 0.02 & 0.02 & 0.16 & 0.02 \\
\hline 34 & $4: 30: 49.86$ & $64: 50: 55.7$ & 0.27 & 0.56 & -8.88 & -1.25 & 0.30 & -8.89 & -0.87 & 0.31 \\
\hline & & & & & 0.01 & 0.04 & 0.01 & 0.01 & 0.04 & 0.01 \\
\hline 35 & $4: 30: 49.86$ & $64: 50: 50.6$ & 0.55 & 0.56 & -10.23 & 0.22 & 0.52 & -10.24 & 0.41 & 0.53 \\
\hline & & & & & 0.01 & 0.06 & 0.01 & 0.01 & 0.06 & 0.01 \\
\hline 36 & 4:30:51.03 & $64: 50: 49.2$ & 0.27 & 0.56 & -8.64 & -1.67 & -.19 & -8.63 & -1.06 & -0.19 \\
\hline
\end{tabular}


TABLE 2-Continued

\begin{tabular}{|c|c|c|c|c|c|c|c|c|c|c|}
\hline Cluster & RA (2000) & DEC (2000) & $\begin{array}{c}\text { Radius } \\
\left({ }^{\prime \prime}\right)\end{array}$ & $\mathrm{E}(\mathrm{B}-\mathrm{V})_{t}$ & $\mathrm{M}_{F 555 W}$ & $(\mathrm{~F} 336 \mathrm{~W}-\mathrm{F} 555 \mathrm{~W})_{0}$ & $(\mathrm{~F} 555 \mathrm{~W}-\mathrm{F} 814 \mathrm{~W})_{0}$ & $\mathrm{M}_{V}$ & $(U-V)_{0}$ & $(\mathrm{~V}-\mathrm{I})_{0}$ \\
\hline \multirow{2}{*}{37} & \multirow{2}{*}{ 4:30:51.09 } & \multirow{2}{*}{$64: 50: 46.9$} & \multirow{2}{*}{0.34} & \multirow{2}{*}{0.56} & 0.01 & 0.04 & 0.03 & 0.01 & 0.04 & 0.03 \\
\hline & & & & & 0.01 & 0.02 & 0.01 & 0.01 & 0.02 & 0.01 \\
\hline \multirow[t]{2}{*}{38} & \multirow[t]{2}{*}{$4: 30: 42.83$} & \multirow[t]{2}{*}{$64: 51: 20.1$} & \multirow[t]{2}{*}{0.60} & \multirow[t]{2}{*}{0.56} & -8.27 & -0.63 & 0.36 & -8.28 & -0.32 & 0.37 \\
\hline & & & & & 0.01 & 0.10 & 0.02 & 0.01 & 0.10 & 0.02 \\
\hline 39 & $4: 30: 51.45$ & $64: 50: 48.8$ & 0.90 & 0.63 & -10.56 & -1.50 & 0.06 & -10.56 & -1.01 & 0.07 \\
\hline \multirow[t]{2}{*}{40} & \multirow[t]{2}{*}{$4: 30: 51.51$} & \multirow[t]{2}{*}{$64: 50: 47.5$} & \multirow{2}{*}{0.80} & \multirow[t]{2}{*}{0.63} & -10.22 & -1.54 & 0.27 & -10.23 & -1.13 & 0.28 \\
\hline & & & & & 0.02 & 0.04 & 0.03 & 0.02 & 0.04 & 0.03 \\
\hline \multirow[t]{2}{*}{41} & \multirow{2}{*}{$4: 30: 51.90$} & \multirow{2}{*}{$64: 50: 47.7$} & \multirow{2}{*}{0.60} & \multirow[t]{2}{*}{0.71} & -9.61 & -0.75 & 0.32 & -9.62 & -0.42 & 0.33 \\
\hline & & & & & 0.02 & 0.09 & 0.03 & 0.02 & 0.09 & 0.03 \\
\hline \multirow[t]{2}{*}{42} & \multirow{2}{*}{$4: 30: 53.34$} & \multirow{2}{*}{$64: 50: 46.2$} & \multirow{2}{*}{0.80} & \multirow[t]{2}{*}{0.63} & -8.93 & 1.12 & 0.27 & -8.94 & 1.29 & 0.28 \\
\hline & & & & & 0.03 & 0.65 & 0.05 & 0.03 & 0.65 & 0.05 \\
\hline \multirow[t]{2}{*}{43} & $4: 30: 53.80$ & $64: 50: 50.4$ & 0.50 & 0.56 & -7.31 & -0.56 & 0.61 & -7.33 & -0.31 & 0.62 \\
\hline & & & & & 0.03 & 0.19 & 0.05 & 0.03 & 0.19 & 0.05 \\
\hline 44 & $4: 30: 54.15$ & $64: 50: 29.5$ & 0.50 & 0.71 & -7.95 & -2.07 & 0.41 & -7.97 & -1.64 & 0.43 \\
\hline & & & & & 0.04 & 2.78 & 0.07 & 0.04 & 2.78 & 0.07 \\
\hline 46 & $4: 30: 55.12$ & $64: 50: 42.9$ & 0.60 & 0.56 & -7.65 & -0.35 & 0.37 & -7.66 & -0.07 & 0.38 \\
\hline & & & & & 0.02 & 0.22 & 0.04 & 0.02 & 0.22 & 0.04 \\
\hline 47 & $4: 30: 55.97$ & $64: 50: 30.3$ & 0.60 & 0.56 & -8.72 & -1.42 & -.10 & -8.72 & -0.88 & -0.10 \\
\hline & & & & & 0.01 & 0.05 & 0.02 & 0.01 & 0.05 & 0.02 \\
\hline 48 & $4: 30: 59.08$ & $64: 50: 19.5$ & 1.14 & 0.56 & -8.82 & -2.07 & 0.07 & -8.82 & -1.53 & 0.08 \\
\hline & & & & & 0.05 & 0.10 & 0.08 & 0.05 & 0.10 & 0.08 \\
\hline
\end{tabular}

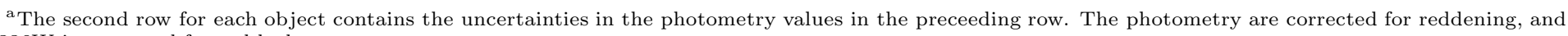
F336W is corrected for red leak. 
This figure "fig10a.jpeg" is available in "jpeg" format from: http://arxiv.org/ps/astro-ph/0009280v1 
This figure "fig10b.jpeg" is available in "jpeg" format from: http://arxiv.org/ps/astro-ph/0009280v1 
This figure "fig11.jpeg" is available in "jpeg" format from: http://arxiv.org/ps/astro-ph/0009280v1 